\title{
ESTUDIO DEL EQUILIBRIO SÓLIDO-LÍQUIDO DE MEZCLAS BINARIAS FORMADAS POR MIRISTATO DE ETILO Y COMPUESTOS REPRESENTATIVOS DEL DIESEL DE PETRÓLEO
}

\author{
María D. ROBUSTILLO-FUENTES ${ }^{1}$, Mariana C. DA COSTA ${ }^{2}$, Antonio J. A. MEIRELLES ${ }^{3}$, \\ Pedro A. PESSÔA FILHO ${ }^{1}$ \\ ${ }^{1}$ Universidad de São Paulo, Escuela Politécnica, Departamento de Ingeniería Química \\ ${ }^{2}$ Universidad de Campinas, Escuela de Ciencias Aplicadas \\ ${ }^{3}$ Universidad de Campinas, Facultad de Ingenieria de Alimentos, Departamento de Ingeniería de \\ Alimentos \\ E-mail de contacto: mrobustillof@gmail.com
}

\begin{abstract}
Biodiesel is becoming a sustainable alternative to fuels derived from petroleum. It is basically composed by fatty acid alkyl esters obtained by reaction between fatty acids and alcohol in an alkaline medium. A promising source of fatty acids in Brazil is the coconut, which could make the process more economical, because it contains myristic acid at very high levels. Biodiesel is usually blended with conventional hydrocarbon-based diesel to avoid flow assurance problems. The reliable prediction of solid liquid equilibrium (SLE) would help to formulate mixtures with the desired cold properties but experimental data to check predictions are scarce. In this work, binary mixtures of ethyl myristate and various petroleum diesel components were analyzed by differential scanning calorimetry in order to obtain experimental data of SLE. Results show the immiscibility of solid phase in all cases and reveal the existence of congruent fusion points never observed before for this kind of systems.
\end{abstract}

\section{INTRODUCCIÓN}

El biodiesel es una mezcla líquida de ésteres de ácidos grasos obtenidos por reacción entre grasas y/o aceites y un alcohol en un medio alcalino, cuya producción viene siendo estimulada en los últimos años debido a razones económicas y a la legislación ambiental. El comportamiento de fase del biodiesel a bajas temperaturas depende de los aceites y/o grasas utilizados en su producción y del alcohol a partir del cual se forma. La mayoría de las fuentes de grasa y vegetales utilizadas para la producción de biodiesel tienen cantidades más significativas de ácido esteárico (C18:0), oleico (C18:1) y linoleico (C18:2); aceite de palma, que contiene ácido palmítico (C16:0) en su mayoría, y el coco, con un predominio de ácidos láurico (C12:0) y mirístico (C14:0), son excepciones. Sin embargo, en Brasil la existencia de coco (Cocos nucifera L.) en abundancia permitiría usarlo como materia prima para la producción de biocombustibles ayudando al desarrollo social y económico del país. Además se trata de una planta que puede ser cultivada en lugares donde otros cultivos no 


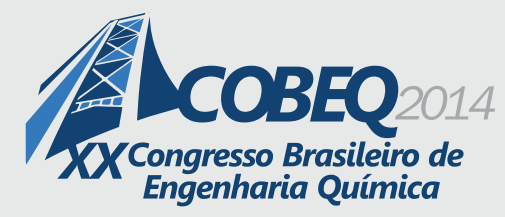

podrían desarrollarse de manera sostenible y tiene um gran potencial para la producción de óleo.

Sin embargo, uno de los problemas del uso de biodiesel a bajas temperaturas se debe a la aparición de fases sólidas, que pueden obstruir los filtros en los vehículos diésel causando daños en el motor. Este hecho puede ser especialmente crítico en el caso del biodiesel producido a partir de etanol, procedimiento poco utilizado o estudiado hasta ahora, aunque prometedor para la industria y economía brasileña.

El comportamiento en frío del biodiesel no debe suponer un problema si, en lugar de utilizarse puro, los motores se alimentan con mezclas de combustible diesel y biodiesel. La adición de biodiesel al combustible diésel tradicional aporta además más lubricidad y reduce las emisiones de partículas finas y óxidos de nitrógeno del vehículo. La composición de las mezclas comercializadas con esas características se designa mediante el factor "B" que indica la cantidad de biodiesel. Por ejemplo, el combustible que contiene un $20 \%$ de biodiesel está etiquetado como B20 y el biodiésel puro se llama B100. La mezcla B20 se puede usar generalmente en los motores sin necesidad de realizar ninguna modificación. Sin embargo, como comentado anteriormente, el uso de biodiesel puro a bajas temperaturas está limitado por la cristalización de sus componentes.

Los posibles problemas de deposición se pueden anticipar si se conocen las principales variables que intervienen en el proceso. Dichas variables son principalmente el punto de niebla (CP), que corresponde a la temperatura a la que comienza a observarse la formación de cristales; punto de obstrucción del filtro en frío (CFPP), temperatura a la que comienza el bloqueo del filtro de combustible; y el punto de vertido, que corresponde a la temperatura a la cual el combustible deja de fluir. Estas propiedades pueden limitar la cantidad máxima de biodiesel que se añade al diesel de petróleo con el fin de cumplir con la normativa vigente para el combustible.

El modelado termodinámico adecuado de equilibrio sólido-líquido (SLE) permitiría determinar dichas variables y ayudaría a la elección de las materias primas para producir un biodiesel con una composición favorable en ambientes fríos, así como la formulación, transporte y almacenamiento de las mezclas de diesel/biodiesel. Sin embargo, la información experimental disponible en la actualidad para comprobar la fiabilidad de los modelos es escasa.

Algunos autores han analizado compuestos puros individuales y mezclas binarias o ternarias de ésteres de ácidos grasos (Dörfler y Pietschmann (1990), Imahara et al. (2006), Lopes et al. (2008), Boros et al. (2009), Costa et al. (2012), Robustillo et al. (2013 a, b; 2014 a, b). No obstante, pocos diagramas de fase de sistemas formados por ésteres etílicos o metílicos y compuestos representativos del diesel convencional se han encontrado en la bibliografía. Algunas excepciones son los trabajos de Collinet y Gmehling (2005) y Benziane et al. (2013 a, b), donde se estudiaron las mezclas de miristato de etilo y p-xileno, así como mezclas de ésteres metílicos de ácidos grasos con alcanos pesados y aromáticos, respectivamente.

Por esta razón, el objetivo del presente trabajo es el estudio del equilibrio sólido líquido de mezclas binarias de miristato de etilo (éster etílico del ácido mirístico que se encuentra en abundancia en el biodiesel producido a partir de aceite de coco) y compuestos representativos del diesel de 
petróleo, tales como el decano, dodecilciclohexano y p-xileno (Buczynsky, 2009), mediante calorimetría diferencial de barrido.

\section{SECCIÓN EXPERIMENTAL}

Los reactivos utilizados para la preparación de las mezclas (miristato de etilo, n-decano, dodecilciclohexano y p-xileno) se muestran en la Tabla 1. El calorímetro se calibró con índio, naftaleno, ciclohexano y n-decano. No se realizó ninguna purificación adicional para ninguno de los reactivos. Los análisis se realizaron en un equipo comercializado por Mettler Toledo modelo DSC822e. Las mezclas $(5-7 \mathrm{mg})$ se prepararon en una balanza analítica con una precisión de \pm $0,2 \mathrm{mg}$.

Tabla 1 - Propiedades y suministradores de los compuestos utilizados en los experimentos

\begin{tabular}{llcc}
\hline Nombre químico & Suministrador & Pureza & Peso molecular $\left(\mathbf{g} \cdot \mathbf{m o l}^{-1}\right)$ \\
\hline Miristato de etilo & Sigma Aldrich & $\geq 0,98$ & 256,42 \\
Dodecilciclohexano & TCI America & $\geq 0,98$ & 252,49 \\
p-xileno & Sigma Aldrich & $\geq 0,99$ & 106,17 \\
Indio $^{*}$ & TA Instruments & 0,9999 & 114,80 \\
Naftaleno $^{*}$ & Merck & $\geq 0,99$ & 128,17 \\
Ciclohexano $^{*}$ & Merck & $\geq 0,99$ & 84,16 \\
n-decano & Sigma Aldrich & $\geq 0,99$ & 142,28 \\
\hline
\end{tabular}

* Compuestos utilizados para calibrar el equipo de DSC.

El procedimiento utilizado para obtener el perfil de temperatura mediante calorimetría diferencial de barrido fue el mismo utilizado en trabajos anteriores [2]; brevemente:

(1) Se pesaron entre 5 y $7 \mathrm{mg}$ de cada mezcla binaria y se colocaron en crisoles de aluminio herméticos.

(2) La muestra se calentó hasta aproximadamente $15 \mathrm{~K}$ por encima de la temperatura de fusión del compuesto puro com mayor temperatura de fusión.

(3) La muestra se enfrió (a una velocidad de enfriamiento de $1 \mathrm{~K} \cdot \min ^{-1}$ ) hasta $25 \mathrm{~K}$ por debajo de la temperatura del compuesto puro de menor temperatura de fusión y se mantuvo constante a dicha temperatura durante 10 minutos.

(4) Se calentó de nuevo la muestra (también a una velocidad de calentamiento de $1 \mathrm{~K}$. $\min ^{-1}$ ) hasta que se haya producido la fusión completa.

El flujo de nitrógeno utilizado fue de $50 \mathrm{ml} \cdot \min ^{-1}$ y la pureza del mismo $99,99 \%$. Como criterio general, se consideró que la temperatura de fusión es la correspondiente al pico observado a la temperatura más alta y que a su vez se corresponde con el valor mínimo absoluto del flujo de calor en el termograma de calentamiento. 


\section{RESULTADOS}

Las temperaturas de fusión de los compuestos puros determinadas en este trabajo mediante calorimetría diferencial de barrido se muestran en la Tabla 2.

Tabla 2 - Temperaturas de fusión de los compuestos puros

\begin{tabular}{lc}
\hline Nombre químico & $\mathbf{T}_{\text {fusión }}(\mathbf{K})$ \\
\hline Miristato de etilo (1) & 287.32 \\
n-decano (2) & 244.54 \\
Dodecilciclohexano (3) & 286.47 \\
p-xileno (4) & 286.73 \\
\hline
\end{tabular}

Los termogramas de los sistemas binarios estudiados se muestran en las Figuras 1 y $2 \mathrm{ab}$.
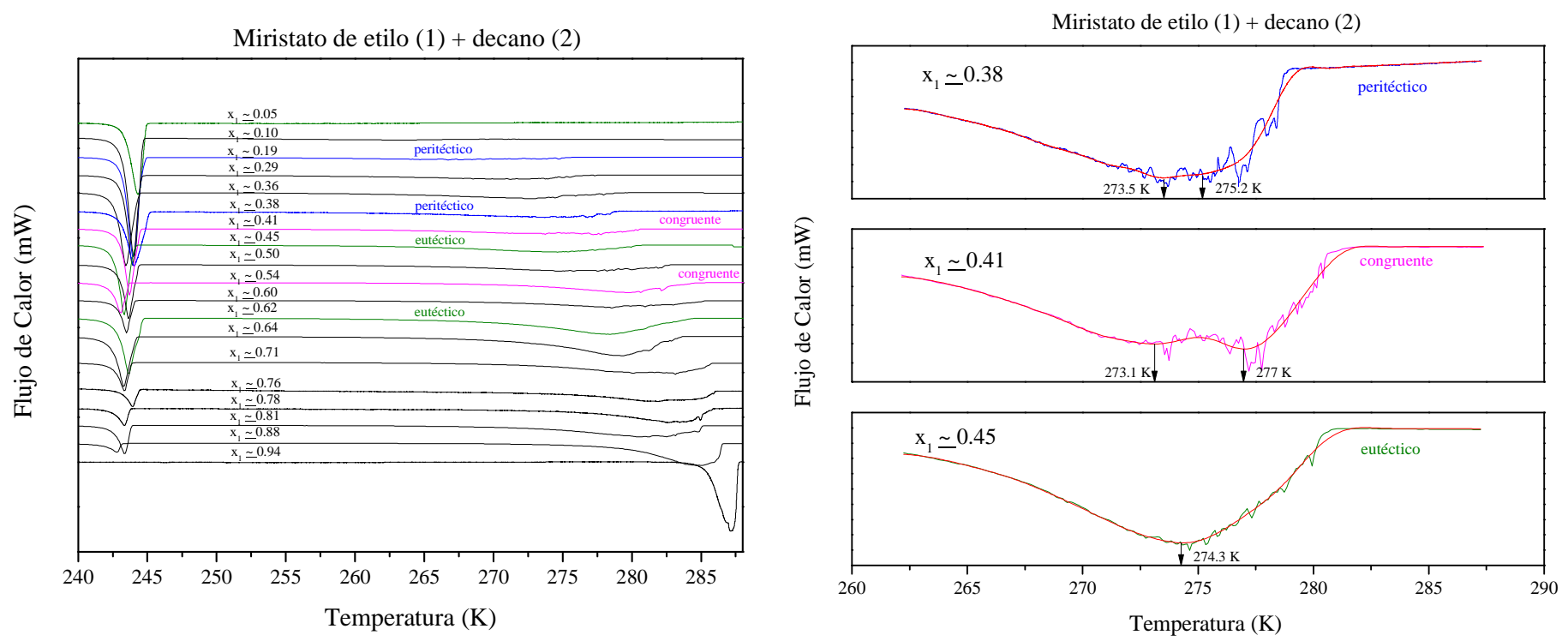

Figura 1 - Termogramas del sistema binario formado por miristato de etilo y decano: a) termogramas completos (izquierda); b) ampliación del pico superior para algunas composiciones (derecha).

En los termogramas del sistema miristato de etilo + decano (Figura 1), se observa, a la misma temperatura para todas las composiciones y a excepción de la de $95 \%$ de miristato de etilo, un pico nítido localizado en la parte izquierda de los termogramas, que correspode a la transición eutéctica. El hecho de que ese pico desaparezca para la composición del $95 \%$ de miristato de etilo indica que esa concentración corresponde a una región de miscibilidad parcial. La intensidad de este pico va disminuyendo a medida que aumenta la concentración de miristato de etilo y disminuye la de decano (compuesto de menor temperatura de fusión en estado puro). 


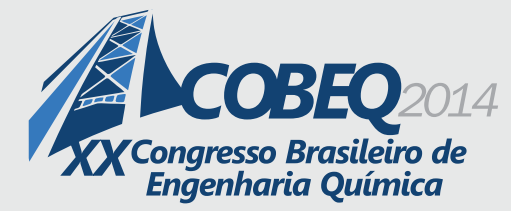

El pico que se encuentra más hacia la derecha se atribuye a la fusión de la mezcla (línea de líquidus) y en general disminuye al reducir la concentración del compuesto con mayor temperatura de fusión (miristato de etilo). En algunos casos, como para la concentración de 0.45 molar de miristato de etilo, se produce la tendencia inversa, lo que se debe a la aparición de un compuesto de fusión congruente, es decir, un compuesto que funde a una temperatura determinada para dar un líquido de la misma composición. Adicionalmente se observan a su vez pequeñas transiciones en los picos superiores que probablemente corresponden a eutécticos y peritécticos locales.

En los termogramas de las mezclas de miristato de etilo com n-decano se observan un mayor número de señales que para los termogramas correspondientes a las mezclas de miristato de etilo con dodecilciclohexano o p-xileno (Figura 2a y b, respectivamente). No obstante, algunas de ellas no son más que ruido o señales generadas por problemas de miscibilidad en la fase líquida, por lo que fue necesario utilizar una función basada en la Transformada de Fourier para eliminar el ruido antes de determinar las temperaturas de transición, como se puede observar en la Figura $1 \mathrm{~b}$ mostrada anteriormente.
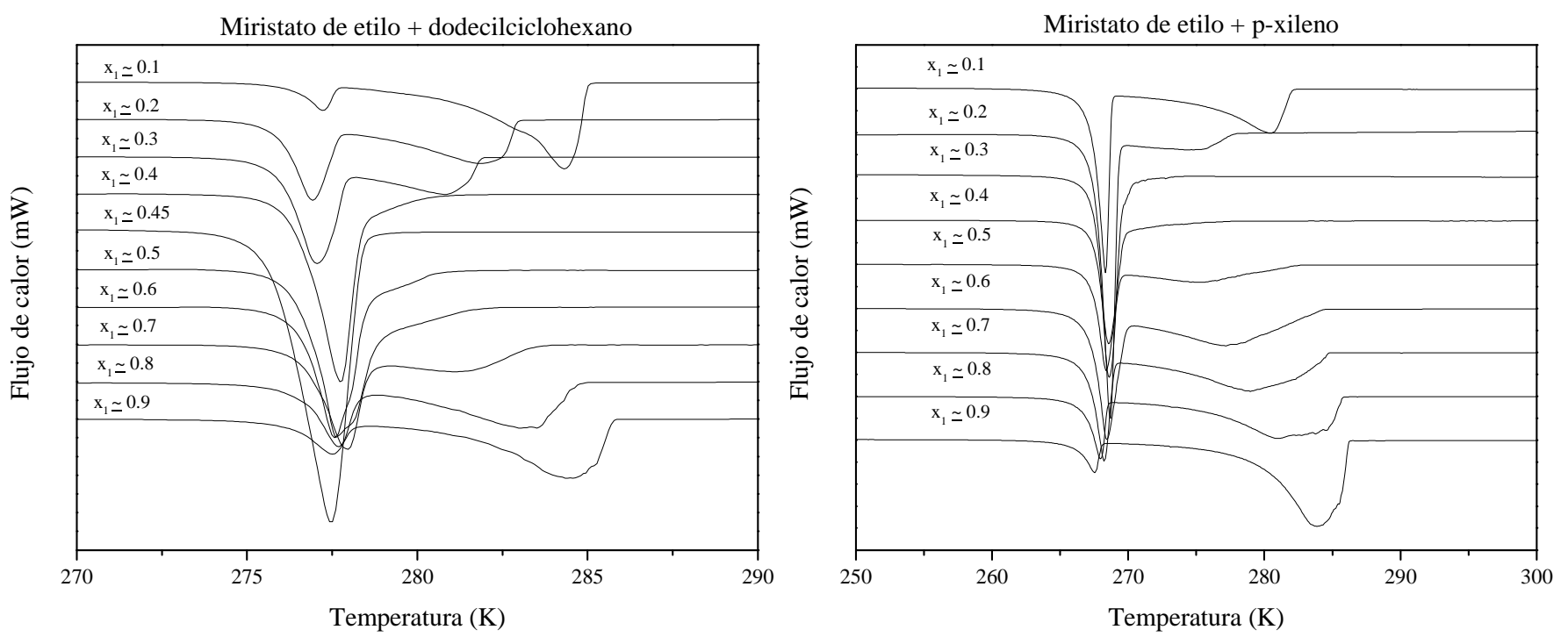

Figura 2 - Termogramas de los sistemas binarios formados por miristato de etilo y dodecilciclohexano (izquierda (a)); miristato de etilo y p-xileno (derecha (b)).

Los diagramas de fases de los sistemas binarios estudiados en este trabajo se muestran en la Figura 3. Las posibles fases sólidas existentes se representan con letras griegas. Los triángulos observados en la Figura $3 \mathrm{c}$ representan los datos experimentales previamente obtenidos por Collinet y Gemehlig (2005). Los resultados obtenidos por dichos autores son acordes con los resultados obtenidos en este trabajo. Pequeñas diferencias pueden ser debidas al empleo de técnicas diferentes, puesto que Collinet y Gemehlig utilizaron la microscopía en lugar de la calorimetría diferencial de barrido. Los resultados de la microscopía parecen ser un poco mayores 


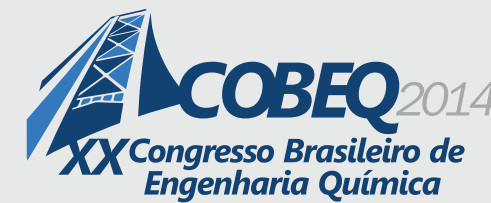

19 a 22 de outubro de 2014

Florianópolis/SC

que los de DSC, lo que concuerda con el hecho de que para detectar la aparición o desaparición de cristales por microscopia puede ser necesaria una mayor cantidad de muestra y el ojo humano influye en los resultados y los aporta un mayor caracter subjetivo. Para los otros dos sistemas binarios no se han encontrado datos en la bibliografia con los cuales se puedan comparar los resultados obtenidos en el presente estudio.
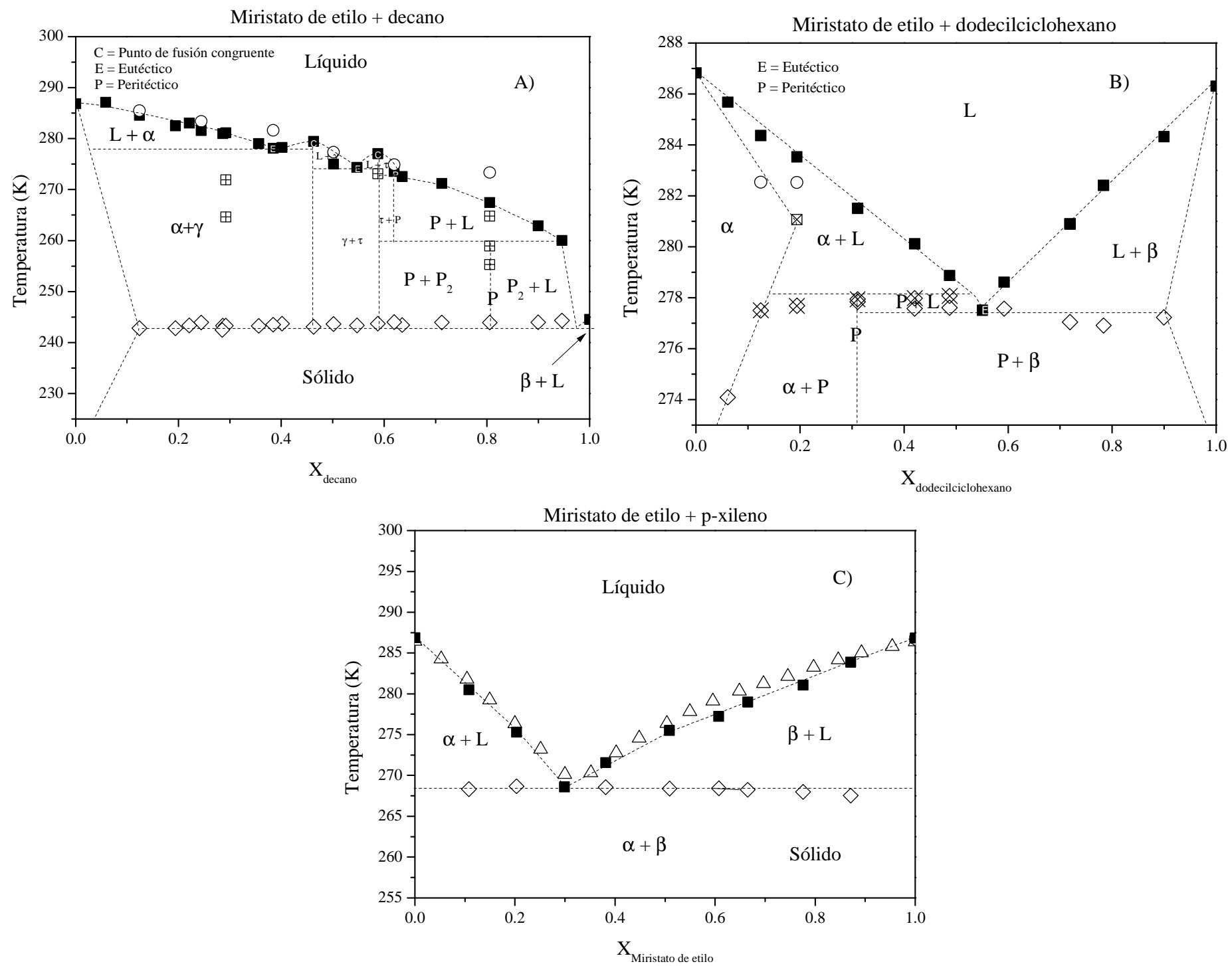

Figura 3 - Diagramas de fase propuestos para los sistemas estudiados.

Como se puede observar, todos los sistemas presentan un punto eutéctico. No obstante, los sistemas que contienen decano y dodecilciclohexano presentan a su vez puntos peritécticos. El sistema formado por miristato de etilo y dodecilciclohexano es aún más complejo y presenta 


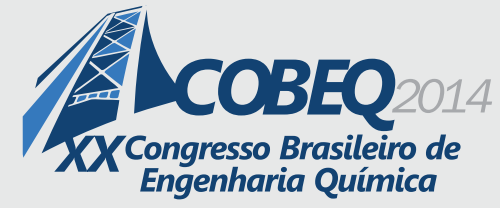

dos puntos de fusión congruente; este tipo de transición no habia sido observada anteriormente en sistemas que contienen ésteres de ácidos grasos.

\section{CONCLUSIONES}

En este trabajo se determinaron experimentalmente los datos de equilibrio sólido líquido de sistemas binarios formados por miristato de etilo y tres compuestos diferentes que pueden estar presentes en el diesel convencional (una parafina lineal (n-decano), un nafteno (dodecilciclohexano) y un compuesto aromático (p-xileno). Todos los sistemas presentan inmiscibilidad en la fase sólida. La complejidad de estos sistemas, especialmente los formados por miristato de etilo con decano o dodecilciclohexano - este último presenta además puntos de fusión congruentes-, puede tener impactos inesperados en el comportamiento del biodiesel a bajas temperaturas y su conocimiento resulta necesario para poder formular mezclas de biodiesel/diesel adecuadas y evitar problemas durante el transporte y uso del combustible.

\section{AGRADECIMIENTOS}

Los autores agradecen a FAPESP (procesos 2010/18355-1, 2008/56258-8 y 2012/05027-1), CAPES y CNPq por la financiación recibida para el desarrollo de este trabajo, así como a los miembros de los laboratorios ExTrAE (UNICAMP), Síntesis y Caracterización de Polímeros (CQMA / IPEN) y GenBio (USP) por la disponibilidad del equipo de DSC y la ayuda prestada en la realización de los experimentos.

\section{REFERENCIAS BIBLIOGRÁFICAS}

BENZIANE, M.; KHIMECHE, K.; DAHMANI, A.; NEZAR, S.; TRACHE, D. Experimental determination and prediction of (solid plus liquid) phase equilibria for binary mixtures of heavy alkanes and fatty acids methyl esters. J Therm. Anal. Calorim., v. 112, p. 229-235, 2013.

BENZIANE, M.; KHIMECHE, K.; TRACHE, D.; DAHMANI, A. Experimental determination and prediction of (solid + liquid) phase equilibria for binary mixtures of aromatic and fatty acids methyl esters. J. Therm. Anal. Calorim., v. 114, p. 1383-1389, 2013.

BOROS, L.; BATISTA, M. L. S.; VAZ, R. V.; FIGUEIREDO, B. R.; FERNANDES, V. F. S.; COSTA, M. C.; KRÄHENBÜHL, M. A.; MEIRELlES, A. J. A.; COUTINHO, J. A. P. Crystallization behavior of mixtures of fatty acid ethyl esters with ethyl stearate. Energy \& Fuels, v. 23, p. 4625-4629, 2009.

BUCZYNSKY, A. E. US Patent 2010/0287819 A1. 2009.

COLLINET, E.; GMEHLING, J. Activity coefficient at infinite dilution, azeotropic data, excess enthalpies and solid-liquid-equilibria for binary systems of alkanes and aromatics with esters. Fluid Phase Equilib. v. 230/1-2, p. 131-142, 2005. 
COSTA M. C.; BOROS L. A. D.; BATISTA M.L.S.; COUTINHO J.A.P.; KRÄHENBÜHL M.A.; MEIRELLES A.J.A. Phase diagrams of mixtures of ethyl palmitate with fatty acid ethyl esters. Fuel, v. 91, p. 177-181, 2012.

DÖRFLER, H.-D.; PIETSCHMANN, N. Phase and miscibility behavior of binary lipid systems. Colloid Polym. Sci., v. 268, p. 567-577, 1990.

IMAHARA, H.; MINAMI, E.; SAKA, S. Thermodynamic study on cloud point of biodiesel with its fatty acid composition. Fuel, v. 85, p. 1666-1670, 2006.

LOPES, J. C. A.; BOROS, L.; KRÄHENBÜHL, M. A.; MEIRELLES, A. J. A.; DARIDON, J. L.; PAULY, J.; MARRUCHO, I. M.; COUTINHO, J. A. P. Prediction of cloud points of biodiesel, Energy \& Fuels, v. 22, p. 747-752, 2008.

ROBUSTILlO, M. D.; BARBOSA, D.; MEIRELleS, A. J. A.; PESSOA FILHO, P. A. Solid-liquid equilibrium in ternary mixtures of ethyl oleate, ethyl laurate and ethyl palmitate. Fluid Phase Equilib., v. 339, p. 58-66, 2013a.

ROBUSTILlO, M. D.; BARBOSA, D.; MEIRELleS, A. J. A.; PESSOA FILHO, P. A. Solid-liquid equilibrium in ternary mixtures of ethyl laurate, ethyl palmitate and ethyl stearate. Fluid Phase Equilib., v. 358, p. 272-281, 2013b.

ROBUSTILlO, M. D.; BARBOSA, D.; MEIRELleS, A. J. A.; PESSOA FILHO, P. A. Solid-liquid equilibrium in ternary mixtures of ethyl laurate, ethyl palmitate and ethyl myristate. Fluid Phase Equilib., v. 361, p. 188, 2014a.

ROBUSTILlO, M. D.; BARBOSA, D.; MEIRELleS, A. J. A.; PESSOA FILHO, P. A. Solid-liquid equilibrium in ternary mixtures of ethyl oleate, ethyl myristate and ethyl stearate. Fluid Phase Equilib., v. 370, p. 85-94, 2014b. 